\title{
Ocorrência de infecção Cryptosporidium spp. em peixe-boi marinho (Trichechus manatus)
}

\author{
Occurrence of Cryptosporidium spp. infection in antillean manatee (Trichechus manatus) \\ João Carlos Gomes Borges ${ }^{1 *}$; Leucio Câmara Alves²; Jociery Einhardt Vergara-Parente ${ }^{3}$; \\ Maria Aparecida da Glória Faustino²; Erilane de Castro Lima Machado ${ }^{4}$
}

${ }^{1}$ Projeto Peixe-Boi, Fundação Mamíferos Aquáticos, Ilha de Itamaracá - PE, Brasil

${ }^{2}$ Laboratório de Doenças Parasitárias dos Animais Domésticos, Departamento de Medicina Veterinária, Universidade Federal Rural de Pernambuco - UFRPE

${ }^{3}$ Fundação Mamíferos Aquáticos, Recife - PE, Brasil

${ }^{4}$ Departamento de Nutrição, Universidade Federal de Pernambuco - UFPE

Recebido em 2 de Agosto de 2007

Aceito em 22 de Fevereiro de 2009

\section{Resumo}

A criptosporidiose constitui-se como uma zoonose que pode afetar o homem e uma ampla variedade de animais domésticos e silvestres, principalmente indivíduos imunodeficientes. O objetivo desse trabalho foi registrar a ocorrência de infecção por Cryptosporidium em peixe-boi marinho. Após ser constatada a mudança de comportamento de um peixe-boi marinho mantido nos oceanários do Centro Mamíferos Aquáticos, ICMBio - FMA, animal foi submetido à exame clínico e, posteriormente, à coleta de amostra fecal. As amostras fecais foram analisadas pela técnica de Kinyoun, teste de imunofluorescência direta e pelo corante 4'.6'-Diamidino-2-Phenilindole (DAPI). No exame clínico, o animal apresentou sinais de desconforto abdominal. Os resultados obtidos nas análises de microscopia de luz e fluorescente revelaram a presença de oocistos de Cryptosporidium nas fezes desse peixe-boi.

Palavras-chave: Cryptosporidium, região Nordeste, mamíferos aquáticos, sirênios.

\begin{abstract}
Cryptosporidiosis is a zoonosis which can affect man and a wide range of domestic and wild animals, mainly immunodeficient individuals. The objective of this paper was reported the occurrence of a Cryptosporidium infection in Antillean manatee. After an unusual behavior of an Antillean manatee kept in captivity at the Centro Mamíferos Aquáticos, ICMBio - FMA, clinical examination and posterior fecal sampling was performed. Fecal samples were examined by the Kinyoun technique, Direct Immunofluorescence Test and also examined by 4'.6'-Diamidino-2-Phenylindole (DAPI) staining. At the clinical examination, the animal showed signs of abdominal pain. The results obtained by light and fluorescence microscopy analysis showed the presence of Cryptosporidium spp. oocyst in feces of this manatee.
\end{abstract}

Keywords: Cryptosporidium, Northeastern Region, aquatic mammals, sirenians.

Os peixes-boi marinhos (Trichechus manatus) são mamíferos aquáticos de hábitos exclusivamente herbívoros, que habitam áreas costeiras brasileiras e estuarinas, sendo distribuídos de forma descontínua entre os estados de Alagoas e Pará (LIMA, 1997).

Entre os agentes parasitários relacionados a infecçóes em Sirênios, pode-se destacar o gênero Cryptosporidium, em dugongos, Dugong dugon (HILL et al., 1997) e em peixe-boi marinho mantido em ambiente natural (MARCONDES et al., 2002). Além desses, a ocorrência de oocistos de Cryptosporidium foi relatada em animais domésticos, silvestres, répteis, peixes e no

*Autor para correspondência: João Carlos Gomes Borges

Projeto Peixe-Boi, Estrada do Forte Orange, s/n, CP 01, CEP 53900-000,

Ilha de Itamaracá - PE, Brasil; e-mail: borges_pb@yahoo.com.br homem (LALLO, 1996, GRACZYK; CRANFIELD, 2000; COX et al., 2005).

Segundo Cox et al. (2005), frequentemente esta enfermidade encontra-se relacionada a hospedeiros imunodeficientes, podendo ocasionar diarréia aguda, dor abdominal, náuseas, vômitos, anorexia e, dependendo da severidade do quadro, pode resultar em morte.

Os oocistos de Cryptosporidium possuem a capacidade de resistir aos desinfetantes comumente utilizados, permanecendo no ambiente durante semanas ou meses, inclusive em diferentes temperaturas e salinidades (FAYER et al., 2004), contribuindo assim para a sua rápida dispersão.

Diante da ocorrência do Cryptosporidium em diferentes espécies de mamíferos aquáticos (DENG et al., 2000; SANTÍM 
et al., 2005), e frente à possibilidade da infecção nos peixes-boi marinhos, o efetivo diagnóstico desse agente torna-se de fundamental importância, no intuito de serem estabelecidas medidas preventivas, visto que essa espécie de mamífero aquático encontra-se seriamente ameaçada de extinçáo (IBAMA, 2001). Sendo assim, este trabalho foi desenvolvido com o objetivo de relatar a ocorrência de Cryptosporidium em peixe-boi marinho (T. manatus) mantido em oceanário.

Entre os animais cativos nos oceanários do Centro Mamíferos Aquáticos, ICMBio - FMA, localizados na Ilha de Itamaracá, $\mathrm{PE}$, foi coletada amostra fecal de um peixe-boi marinho macho, com 14 anos de idade, $340 \mathrm{~kg}$ e $296 \mathrm{~cm}$ de comprimento total. No momento do exame clínico, o animal apresentou desconforto abdominal, aumento do intervalo respiratório e letargia, sendo administrado o tratamento de suporte à base de óleo mineral (por via oral) e antibioticoterapia ${ }^{1}$ por via intramuscular.

O material fecal foi encaminhado ao Laboratório de Doenças Parasitárias dos Animais Domésticos, Departamento de Medicina Veterinária da Universidade Federal Rural de Pernambuco, sendo, em seguida, submetido à sedimentação pelo formol-éter, com posterior confecção dos esfregaços e coloração através da técnica de Kinyoun (BRASIL, 1996), ao teste de imunofluorescência direta $^{2}$ e ao corante 4'.6'-Diamidino-2-Phenilindole (DAPI).

Foram identificados oocistos de Cryptosporidium no material fecal analisado pela técnica de Kinyoun, e o diagnóstico foi confirmado pelos testes de imunofluorescência direta e DAPI.

Após a administraçáo do tratamento de suporte, o animal foi monitorado, sendo constatado o desaparecimento dos sinais clínicos apresentados com aproximadamente 48 horas. Dessa forma, as manifestaçóes clínicas observadas no animal examinado podem ser sugestivas de transtornos ocasionados por Cryptosporidium ou ainda a sua ação conjunta com outros patógenos constatou-se que o desaparecimento desses transtornos pode ter sido decorrente da açáo da conduta terapêutica empregada ou, ainda, do seu caráter autolimitante (LEVINE et al., 1988; LALLO, 1996).

O diagnóstico da infecçáo por Cryptosporidium, neste estudo, foi possível pela associação dos sinais clínicos e pela utilização de três técnicas distintas de exames laboratoriais, as quais vêm comumente sendo realizadas em outras pesquisas que envolvem outras espécies de mamíferos aquáticos (DENG et al., 2000; SANTÍN et al., 2005).

Vale salientar que o peixe-boi avaliado encontrava-se mantido com outros oito espécimes em uma estrutura de três oceanários. Atualmente, a captação de água desses recintos provém de águas marinhas costeiras e de um poço artesiano situado nas imediaçôes das instalaçôes. Essas águas são submetidas ao tratamento diário à base de cloro e, após o processo de sedimentação das partículas em suspensão, são filtradas e aspiradas.

Mesmo diante dos cuidados no tratamento da água desses oceanários, tendo em vista a ocorrência de oocistos de Cryptosporidium já relatada em diversos mananciais aquáticos (JOHNSON et al., 1995), surge uma preocupação eminente à veiculação hídrica desse agente, pois, de acordo com Carey et al. (2004), a ineficiência dos métodos de tratamento de água permite a sua disseminação por causa da resistência aos desinfetantes

${ }^{1}$ Pentabiótico veterinário pequeno porte $600.000 \mathrm{U}$

${ }^{2}$ Kit Merifluor - Meridian Bioscience Diagnostics, Cincinnatti, Ohio comumente empregados e das limitaçôes das técnicas de filtração, em decorrência do pequeno tamanho dos oocistos.

Avaliando ainda outros fatores que podem ter proporcionado a veiculação dos oocistos de Cryptosporidium ao peixe-boi infectado, destaca-se o contato com pessoas, durante as ocasióes de manejo, visto o seu caráter zoonótico de transmissão (FAYER, 2004), bem como os itens alimentares constituintes da dieta desses animais.

A presença do animal infectado, entre outros peixes-boi cativos, pode favorecer a disseminação da infecção, o que torna oportuna a realização de exames laboratoriais de rotina em todos os animais cativos, com ou sem sinais clínicos. Por outro lado, como há possibilidade de transmissão ao homem, dependendo da espécie de Criptosporidium, o manejo desses animais pela equipe técnica e tratadores pode representar risco de infecção humana. Dessa forma, torna-se imprescindível a caracterização genotípica do material.

\section{Referências}

BRASIL. Ministério da Saúde. Infecçóes oportunistas por parasitas em AIDS: técnicas de diagnóstico. Brasília, 1996. 27 p.

CAREY, C. M.; LEE, H.; TREVORS, J. T. Biology, persistence and detection of Cryptosporidium parvum and Cryptosporidium hominis oocyst. Water Research v. 38, n. 4, p. 818-862, 2004.

COX, M. J.; ELWIN, K.; MASSAD, E.; AZEVEDO, R. S. Age-specific seroprevalence to an immunodominant Cryptosporidium sporozoite antigen in a Brazilian population. Epidemiology and Infection, v. 133, n. 5, p. 951-956, 2005.

DENG, M.; PETERSON, R. P.; CLIVER, D. O. First findings of Cryptosporidium and Giardia in California sea lions (Zalophus californianus). Journal of Parasitology, v. 86, n. 3, p. 490-494, 2000.

FAYER, R. Cryptosporium: a water-borne zoonotic parasite. Veterinary Parasitology, v. 126, n. 1-2, p. 37-56, 2004.

FAYER, R.; DUBEY, J. P.; LINDSAY, D. S. Zoonotic protozoa: from land to sea. Trends in Parasitology, v. 20, n. 11, p. 531-536, 2004.

GRACZYK, T. K.; CRANFIELD, M. Cryptosporidium serpentis oocysts and microsporidian spores in feces of captive snakes. Journal of Parasitology, v. 86, n. 2, p. 413-414, 2000.

HILL, B. D.; FRASER, I. R.; PRIOR, H. C. Cryptosporidium infection in a dugong (Dugong dugon). Australian Veterinary Journal, v. 75, n. 9, p. 670-671, 1997.

INSTITUTO BRASILEIRO DO MEIO AMBIENTE E DOS RECURSOS NATURAIS RENOVÁVEIS - IBAMA. Mamíferos aquáticos do Brasil: plano de ação. 2 ed. Brasília, 2001. 96 p.

JOHNSON, D. C.; REYNOLDS, K. A.; GERBA, C. P.; PEPPER, I. L.; ROSE, J. B. Detection of Giardia and Cryptosporidium in marine waters. Water Science Technology, n. 31, p. 439-442, 1995.

LALLO, M. A. Criptosporidiose canina. Clínica Veterinária, v. 1, n. 2, p. $20-$ 22, 1996

LEVINE, J. F.; LEVY, M. G.; WALKER, R. L. Cryptosporidiosis in veterinary students. Journal of American Veterinary Medical Association, v. 193, n. 11 , p. 1413-1414, 1988.

LIMA, R. P. Peixe-Boi Marinho (Trichechus manatus): distribuiçấo, status de conservação e aspectos tradicionais do litoral nordesse do Brasil. 1997. 80 f. Dissertação (Mestrado) - Universidade Federal de Pernambuco, Recife.

MARCONDES, M. C. C.; BRITO, F. L. C.; BORGES, J. C. G.; LIMA, E. C.; ALVES, L. C.; LIMA, R. P. Cryptosporidium sp in Antillean Manatees (Trichechus manatus manatus) in natural captivity, Paraiba state, Brazil. 2002. Florida Marine Mammal Health Conference. Disponível em: <http:// www. vetmed.utl.edu>. Acesso em: 29 de Julho de 2002.

SANTÍN, M.; DIXON, B. R.; FAYER, R. Genetic characterization of Cryptosporidium isolates from ringed seals (Phoca hispida) in Northern Québec, Canada. Journal of Parasitology, v. 91, n. 3, p. 712-716, 2005. 